\title{
Heterogeneity of the Population of Command Neurons in the Lamprey
}

\author{
Pavel V. Zelenin, Sten Grillner, Grigori N. Orlovsky, and Tatiana G. Deliagina \\ The Nobel Institute for Neurophysiology, Department of Neuroscience, Karolinska Institute, SE-171 77, \\ Stockholm, Sweden
}

\begin{abstract}
The effects of signals transmitted from the brain to the spinal locomotor networks by a population of command neurons are determined by specific functional projections of each individual neuron. To reveal these projections, we used a simple vertebrate model, the lamprey, in which responses of the spinal networks to spikes in single reticulospinal axons were detected by using the spike-triggered averaging of the motoneuronal activity. We found that individual neurons exert a uniform effect
\end{abstract}

on the segmental motor output along the whole extent of their axons. Twenty different patterns of effect, that is, combinations of influences on the segmental motoneuron pools, were found. The widespread projections and heterogeneity of the population of command neurons present a basis for formation of different gross motor synergies.

Key words: posture; locomotion; motor synergy; reticulospinal neurons; motoneurons; lamprey
In vertebrates, the main role in initiation and modification of locomotion is played by the reticulospinal (RS) command system, which projects to the spinal networks generating the basic locomotor pattern (Orlovsky et al., 1999). However, attempts for detailed analyses of this system at the network and cellular level were hampered by the large number of neurons constituting the system and the small effect on motor output exerted by individual neurons (Drew et al., 1986). This difficulty was overcome in the present study, first, by using the simpler vertebrate animal model (lamprey) with an RS system consisting of a relatively small number of neurons, and second, by using the technique of synchronous accumulation of network responses to signals from individual RS neurons.

The general structure of the nervous system in the lamprey, as well as the functional organization of its locomotor control system, are both similar to those in higher vertebrates (Nieuwenhuys et al., 1996). The basic pattern of locomotion (swimming) is generated by spinal networks distributed over $\sim 100$ spinal segments (see Fig. 1). Each segment contains an interneuronal circuit participating in the generation of locomotor oscillations (Grillner et al., 1995). Segmental locomotor output is produced by four motoneuron $(\mathrm{MN})$ pools that innervate the dorsal and ventral parts of a myotome on the two sides, respectively (Tretjakoff, 1927; Rovainen, 1979; Wannier et al., 1998). Descending signals to spinal networks are transmitted by RS pathways containing 2000 axons (Nieuwenhuys, 1972; Ronan, 1989; Bussieres, 1994). The RS neurons perform integration of afferent inputs (vestibular, visual, somatosensory) and inputs from other parts of the CNS (Deliagina et al., 1992, 1993, 2000; Deliagina and Fagerstedt, 2000). The commands sent via RS pathways to

Received May 18, 2001; revised July 19, 2001; accepted July 19, 2001

This work was supported by grants to T.G.D. from the Royal Swedish Academy of Sciences (Research Grant for Swedish-Russian scientific cooperation), the Swedish Medical Research Council (no. 11554), and the Curt Nilsson Foundation. We thank Drs. F. Ullén and P. Wallén for critical review of this manuscript.

Correspondence should be addressed to Dr. T. G. Deliagina, The Nobel Institute for Neurophysiology, Department of Neuroscience, Karolinska Institute, SE-17177, Stockholm, Sweden. E-mail: Tatiana.Deliagina@neuro.ki.se.

Copyright (ㄷ) 2001 Society for Neuroscience $0270-6474 / 01 / 217793-11 \$ 15.00 / 0$ the spinal cord initiate locomotion and exert diverse modifying effects on the spinal networks necessary for the control of equilibrium and steering (Grillner et al., 1995; Deliagina and Fagerstedt, 2000; Deliagina et al., 2000). Individual RS neurons affect different classes of spinal neurons (Rovainen, 1974b; Buchanan and Cohen, 1982; Ohta and Grillner, 1989). Earlier experiments have shown that some RS neurons, when stimulated at high frequency, may exert a detectable effect on the output of spinal locomotor networks (Rovainen, 1967; Buchanan and Cohen, 1982). When discharging at normal frequencies, however, only a small group of neurons in lamprey and fish can individually evoke a prominent motor response (Currie and Carlsen, 1987; Liu and Fetcho, 1999).

The aim of the present study was to characterize the effect of individual RS neurons on locomotor output from the four MN pools of each segment along the whole extent of the spinal cord (see Fig. 1). These data are necessary to understand how the spinal locomotor networks are controlled by the population of command neurons.

A brief account of this study has been published previously in abstract form (Zelenin et al., 2000).

\section{MATERIALS AND METHODS}

Experiments were performed on two types of in vitro preparation dissected from adult lampreys (Ichthyomyzon unicuspis). In the first set of experiments $(n=16)$, the brainstem was isolated together with the spinal cord ( $\sim 40$ segments). The preparation was pinned down in a chamber filled with Ringer's solution (see Fig. $2 A$ ), and an agar barrier was built at the level of spinal segments 5-10 to form two pools. Individual RS neurons were recorded from different reticular nuclei [the mesencephalic reticular nucleus (MRN) and the anterior rhombencephalic reticular nucleus (ARRN), middle rhombencephalic reticular nucleus (MRRN), and posterior rhombencephalic reticular nucleus (PRRN)]. Single spikes in a neuron were evoked by positive current pulses (pulse period 100 msec; current up to $20 \mathrm{nA}$ ) passed through the intracellular microelectrode (see Fig. 2A, Stim ME). Arrival of an RS spike to segment 20 was monitored by a surface electrode (see Fig. $2 A, E, S E$ ). Activity of spinal MNs was recorded bilaterally in segment 20 by means of suction electrodes, from the dorsal and ventral branches of ventral roots (VRs).

Experiments of the second set $(n=20)$ were performed on the isolated spinal cord (segments 1-95) (see Fig. 2B). Single spikes in individual RS axons were evoked by positive current pulses (pulse period 


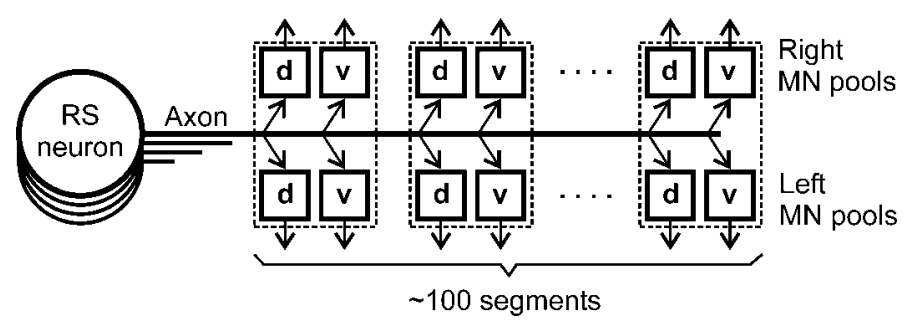

Figure 1. Reticulospinal command system in the lamprey. Individual RS neurons send axons to the spinal cord; many of them reach the most caudal segments. Segmental motor output is generated by four MN pools controlling the dorsal and ventral parts of a myotome on the two sides $(d$ and $v$ pools). The RS neurons may exert their effect on the pools directly or through interneurons.

$150 \mathrm{msec}$; current up to $20 \mathrm{nA}$ ) passed through the intracellular microelectrode (see Fig. 2B, Stim ME). Arrival of a spike to segment 50 was monitored by a surface electrode (see Fig. $2 B, S E$ ). Activity of spinal MNs was recorded from the analogous VR branches at six sites (see Fig. $2 B, D, 1-6)$ along the spinal cord.

To activate the spinal locomotor networks and elicit the neural correlates of locomotion (fictive swimming), D-glutamate (0.5-1 mM) was applied to the spinal cord (Grillner et al., 1981, 1995). The locomotor pattern was characterized by rhythmical bursts of MN activity alternating between the two sides, with in-phase activity in the dorsal and ventral MN pools on each of the sides (see Fig. $2 C$ ) and by a phase lag of the MN activity in the caudal segments relative to the rostral ones (see Fig. 2D).

For each individual RS neuron (axon), a post-RS spike histogram was generated for the spikes of MNs recorded in each VR branch. The time of RS spike occurrence in the cell body (see Fig. $2 A$ ) or at the stimulated site of the axon (see Fig. $2 B$ ) was taken as the origin of the time axis in the histogram (see Fig. $2 F$ ). Typically, responses to a few thousand RS spikes (up to $20 \mathrm{~min}$ of stimulation) were used for generation of a histogram [for details of the technique, see Fetz and Cheney (1980)]. A high-frequency "noise" in the histograms was reduced by "filtering," that is, weighted averaging across seven neighboring bins, as illustrated in Figure $2, F$ and $G$.

To separate activity of individual MNs from the mass activity recorded in a ventral root branch, "sorting of spikes" with DataPac software (Run Technologies) was performed in a few experiments. The criteria for discrimination were the amplitude and width of the positive and negative waveforms (Camp and Pinsker, 1979; Deliagina and Fagerstedt, 2000).

\section{RESULTS}

\section{Types of RS influences on MNs projecting to the same branch}

In the first set of experiments, 60 RS neurons were recorded, and their influences on the spinal motor output were analyzed. Most of them $(n=37)$ were located in the MRRN; the rest were located in the other reticular nuclei. All neurons were larger cells, with axonal conduction velocities ranging from 1.85 to $4.80 \mathrm{~m} / \mathrm{sec}$. Because the neurons from different nuclei were similar in most respects, they will be described together, and a few distinctions among the nuclei will be indicated specifically. The method of RS spike-triggered averaging of the MN activity allowed us to detect influences of individual RS neurons on the spinal motor output, as illustrated in Figure 2G. Influences on at least one ventral root branch were found in the vast majority of RS neurons (MRRN: $34 / 37$, or $92 \%$; MRN: $5 / 6$, or $83 \%$; ARRN: $7 / 7$, or $100 \%$; PRRN: $7 / 10$, or $70 \%$ ).

Four principal types of influence were observed and are illustrated in Figure 3: excitation $(A)$, inhibition $(B)$, excitation followed by inhibition $(C)$, and inhibition followed by excitation $(D)$. "Pure" responses (as in $A$ and $B$ ) were observed much more often than "biphasic" responses (as in $C$ and $D$ ) (67 cases of excitation and 47 of inhibition against 9 of excitation followed by inhibition and 19 of inhibition followed by excitation). The influences could be seen in the ventral root branches both ipsilateral and contralateral to an RS neuron. The amplitude of the response, that is, a deviation of the summated MN activity from the level observed before the arrival of the RS spike, and the response duration varied considerably. For the excitatory responses, the relative amplitude was $120 \pm 140 \%$, and the duration was $18 \pm 12 \mathrm{msec}$ (mean $\pm \mathrm{SD}$ ). For the inhibitory responses, these values were $55 \pm 25 \%$ and $53 \pm 27 \mathrm{msec}$, respectively. A segmental delay of the responses also varied considerably; on average, however, the delay was the shortest for the ipsilateral excitatory responses (Fig. 4A), longer for the ipsilateral inhibitory responses (Fig. $4 B$ ), even longer for the contralateral excitatory responses (Fig. $4 C$ ), and the longest for the contralateral inhibitory responses (Fig. 4D).

Most RS neurons affected the activity of MNs in more than one branch (see Patterns of RS influences on segmental motor input). We compared the segmental delays for responses evoked by a given RS neuron in different branches for three situations: (1) both responses are excitatory, (2) both responses are inhibitory, and (3) one response is excitatory and the other one is inhibitory. We found that influences of the same sign most often had similar segmental delays, and a difference between the two delays was usually $<3$ msec, no matter whether the two branches were located on the same or on the opposite sides of the spinal cord (Fig. $4 E-G$, open and filled circles, respectively).

Do individual RS neurons exert a uniform or diverse action on the MNs projecting through a given branch? We addressed this issue by applying a spike-sorting procedure to the discharges in the branch. Influences of seven RS neurons were analyzed. Figure 5 illustrates such analysis for the RS neuron that exerted an inhibitory action on the whole branch (Fig. 5, all motoneurons). Similar influences were observed in each of the individual MNs separated from the mass activity in the branch (MNs \#1-12). Two other RS neurons evoked an excitatory response in the whole branch. The same type of response was observed in most of the individual MNs projecting to the branch. Finally, four RS neurons evoked biphasic responses in the whole branch. The same type of response was observed in most individual MNs (63\%). In $25 \%$ of MNs, however, no response was detected, whereas $12 \%$ of MNs exhibited only the first or the second component of the response observed in the whole branch. We therefore conclude that individual RS neurons exert a uniform action on the majority of MNs projecting through a given branch. This finding suggests that each of the four MN pools in a segment represents a functional unit.

The types of influences (excitatory, inhibitory, or biphasic) of individual RS neurons on spinal MNs were rather consistent. This was demonstrated first by testing repeatedly a given RS neuron, with the intervals between the tests up to $15 \mathrm{~min}$. For most RS neurons, their effect on MNs was the same in all trials. Only in 4 of 43 RS neurons, with relatively weak effects, did the influences disappeared in one of the trials. A change of the sign of the response was never observed. Second, in three experiments (eight neurons) the effect of concentration of D-glutamate, applied to the spinal cord, was investigated. The change from 0.1 to $5 \mathrm{~mm}$ dramatically affected the level of MN activity: the mean frequency of spikes in the ventral root branches could increase up to 10 times. However, the type of RS influences persisted, whereas the magnitude of the response could change. 
A

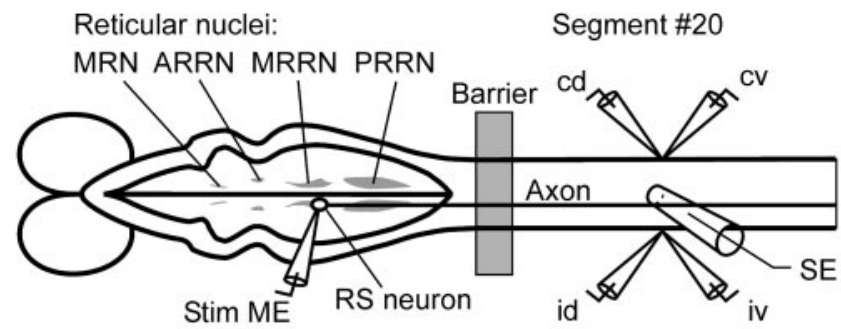

B
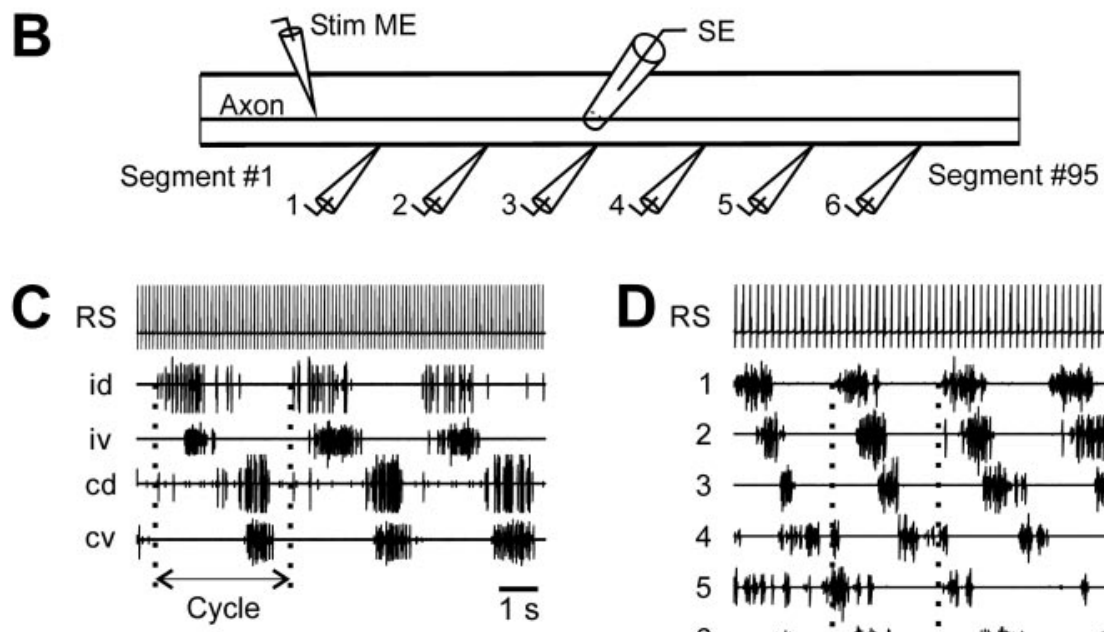

$\mathbf{D}_{\mathrm{RS}}$

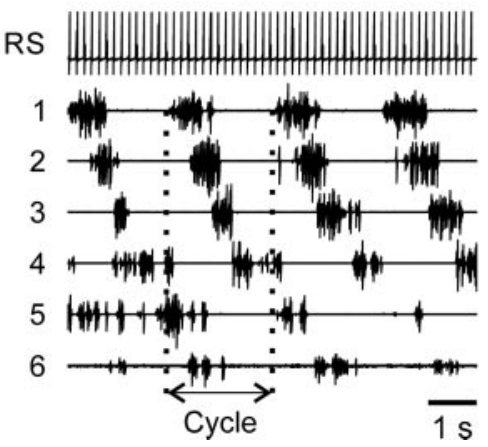

$\mathbf{E}$

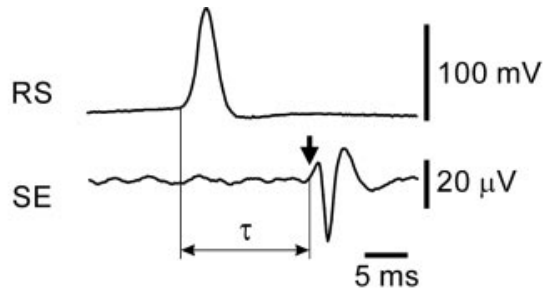

$\mathbf{F}$

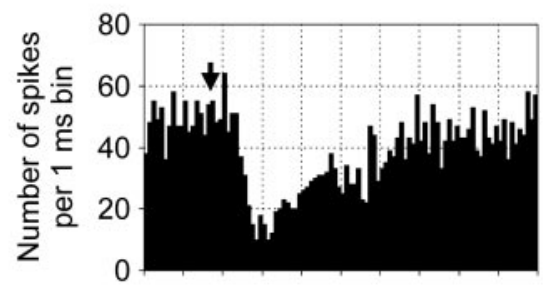

$\mathbf{G}$

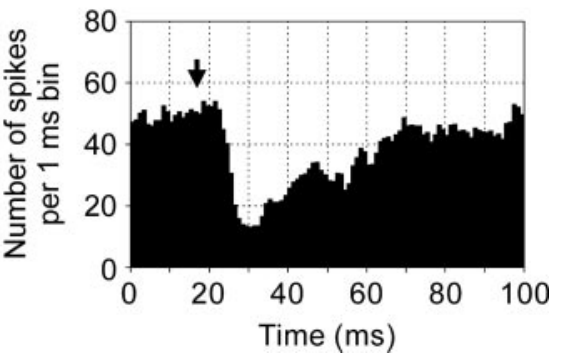

Figure 2. Experimental design and presentation of data. A, Experimental design for studying segmental functional projections of RS neurons. The brainstem and spinal cord were positioned in a chamber divided by a Barrier. The brainstem was positioned in one pool and perfused with Ringer's solution; the spinal cord was positioned in another pool and perfused with Ringer's solution containing D-glutamate to elicit fictive locomotion. Individual reticulospinal neurons (RS neuron) were recorded from different reticular nuclei [the mesencephalic reticular nucleus $(M R N)$ and the anterior rhombencephalic reticular nucleus $(A R R N)$, middle rhombencephalic reticular nucleus $(M R R N)$, and posterior rhombencephalic reticular nucleus $(P R R N)]$. To stimulate a neuron, positive current pulses were passed through the recording intracellular electrode (Stim ME). Activity of MNs was recorded bilaterally in segment 20 by means of suction electrodes from the dorsal and ventral branches of a ventral root (id, ipsilateral dorsal branch; $i v$, ipsilateral ventral; $c d$, contralateral dorsal; $c v$, contralateral ventral). An RS spike arriving at Segment \#20 was recorded by a surface electrode (SE). $B$, Experimental design for studying the longitudinal functional projections of the RS neurons. The spinal cord was positioned in a chamber and perfused with Ringer's solution containing D-glutamate to elicit fictive locomotion. Individual RS axons were stimulated with positive current pulses through the recording intracellular electrode (Stim ME). Activity of MNs was recorded from six analogous ventral root branches along the spinal cord by means of suction electrodes (1-6). An RS spike propagating along the spinal cord was recorded by a surface electrode $(S E)$. $C$, An example of the bursting locomotor-like activity in four ventral root branches evoked by D-glutamate, and the spike activity of the RS neuron evoked by intracellular stimulation. One locomotor Cycle is marked. D, An example of the bursting locomotor-like activity in six analogous ventral root branches evoked by D-glutamate, and the spike activity of the RS neuron evoked by intracellular stimulation. $E$, Recording of the spike in the soma of an RS neuron and in its axon in segment 20. An arrow in this and the following figures indicates an arrival of the RS spike. The time of the spike propagation is designated as $\tau$. $F$, An example of inhibitory influences of an RS neuron revealed by the spike-triggered averaging of 3000 responses. $G$, The same histogram after removal of the high-frequency noise (see Materials and Methods).

\section{Patterns of RS influences on segmental motor output}

The effect of an individual RS neuron on the motor output of one segment can be characterized by a combination of influences on the four branches of the ventral roots of this segment. Different combinations of influences (patterns of segmental response) were observed in different RS neurons. Two of these patterns are illustrated in Figure 6. An RS neuron from MRRN (Fig. 6A) exerted an excitatory influence on the MNs projecting to the ipsilateral ventral branch and inhibitory influences on the MNs projecting to all other branches. An RS neuron from PRRN (Fig. $6 B$ ) excited the ipsilateral "dorsal" and "ventral" MNs and did not influence the contralateral MNs.

Figure 7 summarizes the patterns of segmental response for all 60 recorded RS neurons; for the cases with a biphasic response, only the first phase was considered. Altogether, 20 patterns were observed. We classified these patterns into seven groups accord- ing to their presumed influence on the segmental locomotor output. The basic motor output generated by the spinal locomotor network in the lamprey consists of the alternating bursts of activity of the MNs on the left and right sides; the MNs projecting to the dorsal and ventral parts of a myotome are active in phase (Wannier et al., 1998). In between the bursts the MNs are inhibited (Grillner et al., 1995) and usually do not respond to spikes transmitted by individual RS axons (Fig. $2 C, D$ ). Therefore, the influences of each RS neuron on the left and right sides do not coincide; the phase shift is one-half of the locomotor cycle. These influences should be considered separately. We characterized these influences by two vectors: a vector of body bending when the ipsilateral side is active (the first hemicycle of the locomotor cycle), and a vector of body bending when the contralateral side is active (the second hemicycle). We also assumed that during normal swimming, because of equal activity of dorsal and ventral 

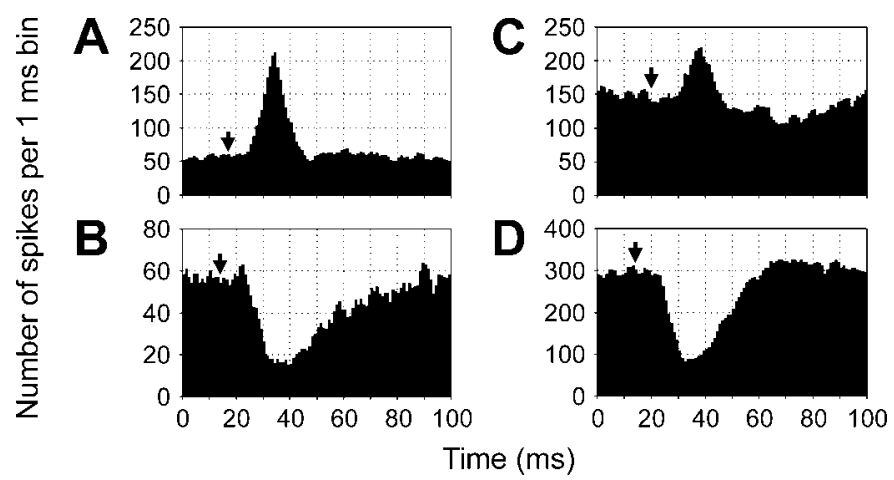

Figure 3. Example of influences of RS neurons on the motor output from segment 20. Each histogram presents the RS spike-triggered averaging of 3000 individual responses, with the bin width of $1 \mathrm{msec}$. The recordings were performed from the ipsilateral dorsal $(A)$, ipsilateral ventral $(B)$, contralateral dorsal $(C)$, and contralateral ventral $(D)$ VR branches. Arrows in $A-D$ indicate the moment of occurrence of RS spike in segment 20.

MNs, both vectors are directed horizontally and are of the same magnitude (Fig. 7, Normal, left column). When the RS neurons were classified, their effect on the direction of body flexion was taken into account first. If the direction did not change, we considered their effects on the magnitude of flexion. The patterns of influences for the seven groups of RS neurons are shown schematically in Figure $7 A-G$ (left column).

Group $A$ (Fig. 7) does not affect the direction of body flexion in any hemicycle but affects symmetrically the magnitude of the ipsilateral and contralateral flexions (denoted by double arrowheads of the vectors). This group includes the neurons that excite (pattern $A 1$ ), inhibit (pattern $A 2$ ), or do not affect (pattern $A 3$ ) the MNs in all four branches.

Group B (Fig. 7) does not affect the directions of body flexion but increases the magnitude of the ipsilateral flexion (denoted by a double arrowhead of the ipsilateral vector). This group includes the neurons that excite both dorsal and ventral MNs on the ipsilateral side (pattern B1), inhibit both dorsal and ventral MNs on the contralateral side (pattern B2), or exert both of these effects (pattern B3).

Group $C$ (Fig. 7) affects the direction of body flexion during both hemicycles by adding a dorsal component of flexion (denoted by an upward turn of both ipsilateral and contralateral vectors). This effect is caused by an increased activity of the dorsal MNs on both sides $(C 1)$, which can be combined with bilateral inhibition of the ventral MNs $(C 2)$.

Group $D$ (Fig. 7) also affects the direction of body flexion on the two sides but in contrast to Group $C$, a ventral component of flexion is added (denoted by a downward turn of both vectors). This effect is caused by inhibition of the dorsal MNs on both sides (D1). This inhibition can be combined with bilateral excitation of the ventral MNs (D2).

Group E (Fig. 7) adds a ventral component to the ipsilateral flexion (denoted by a downward turn of the ipsilateral vector). This effect is caused by an excitation of the ipsilateral ventral MNs (pattern E1) that can be combined with an inhibition of the ipsilateral dorsal MNs (pattern E2). These ipsilateral influences can be combined with different inhibitory influences on the contralateral side (patterns E3-E5). One should notice that the neurons with pattern E5, in contrast to the patterns E1-E4, cause also some ventral flexion on the contralateral side and therefore might be a member of group D. However, the patterns in group
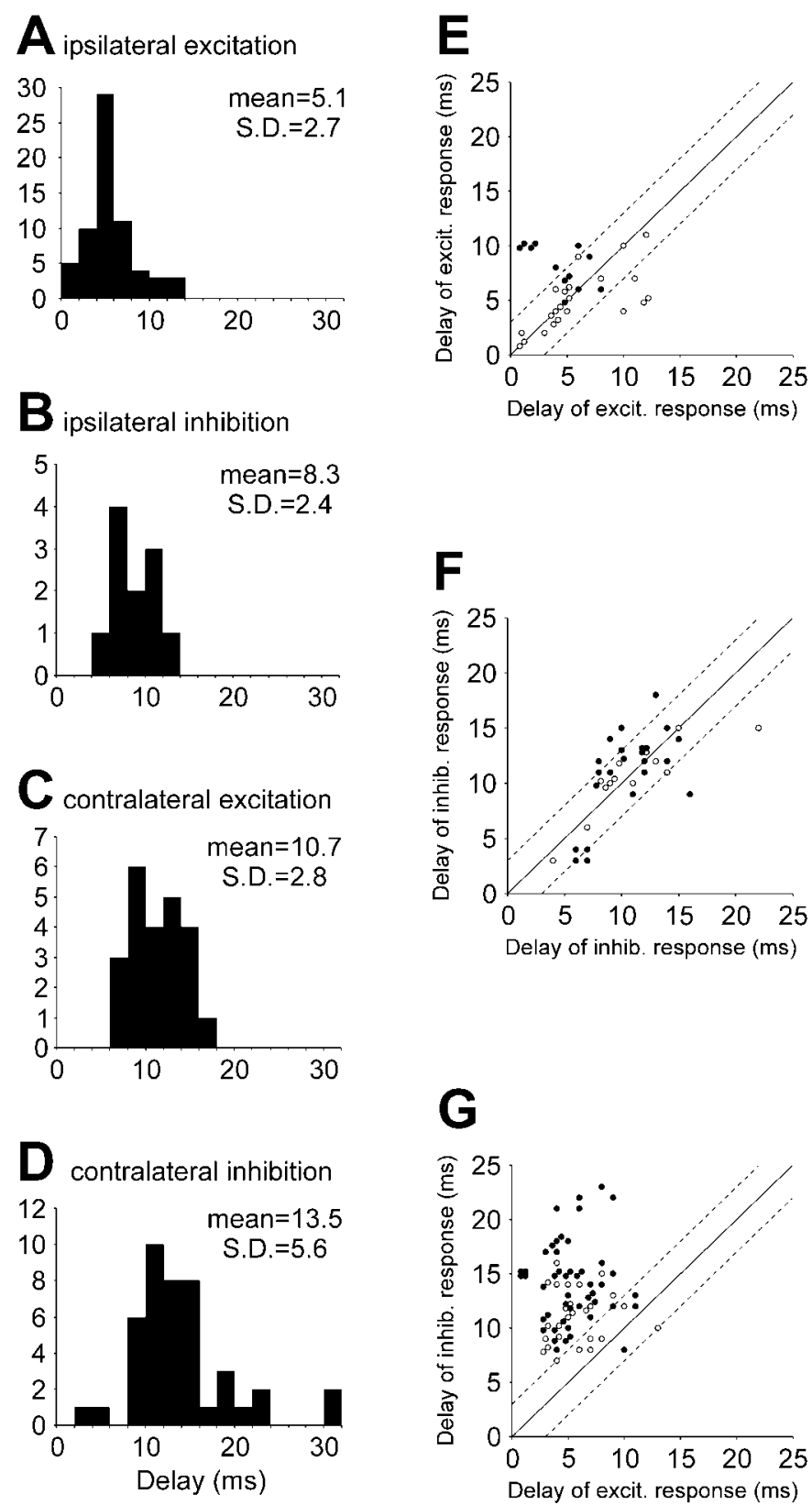

Figure 4. Segmental delays. $A-D$, Segmental delays for different responses: the excitatory ipsilateral $(A)$, inhibitory ipsilateral $(B)$, excitatory contralateral $(C)$, and inhibitory contralateral $(D)$ responses. $E-F$, Segmental delays of responses elicited by the same RS neurons in a pair of ventral root branches. Abscissa, The segmental delay in a branch with the excitatory $(E, G)$ or inhibitory $(F)$ response. Ordinate, The segmental delay in a branch with the excitatory $(E)$ or inhibitory $(F, G)$ response. Open circles represent the pairs of branches located on the same side of the spinal cord; filled circles represent the pairs of branches on the opposite sides. Broken lines demarcate the zone with the difference between the delays in the pair not exceeding $3 \mathrm{msec}$.

D are symmetric, whereas the pattern E5 is a combination of a symmetric pattern D1 with an asymmetric pattern E1. On this basis we included E5 in group E.

Group $F$ (Fig. 7) adds a dorsal component to the contralateral flexion. It includes the pattern F1 with inhibitory influence on the contralateral ventral MNs, and the patterns F2 and F3 in which 

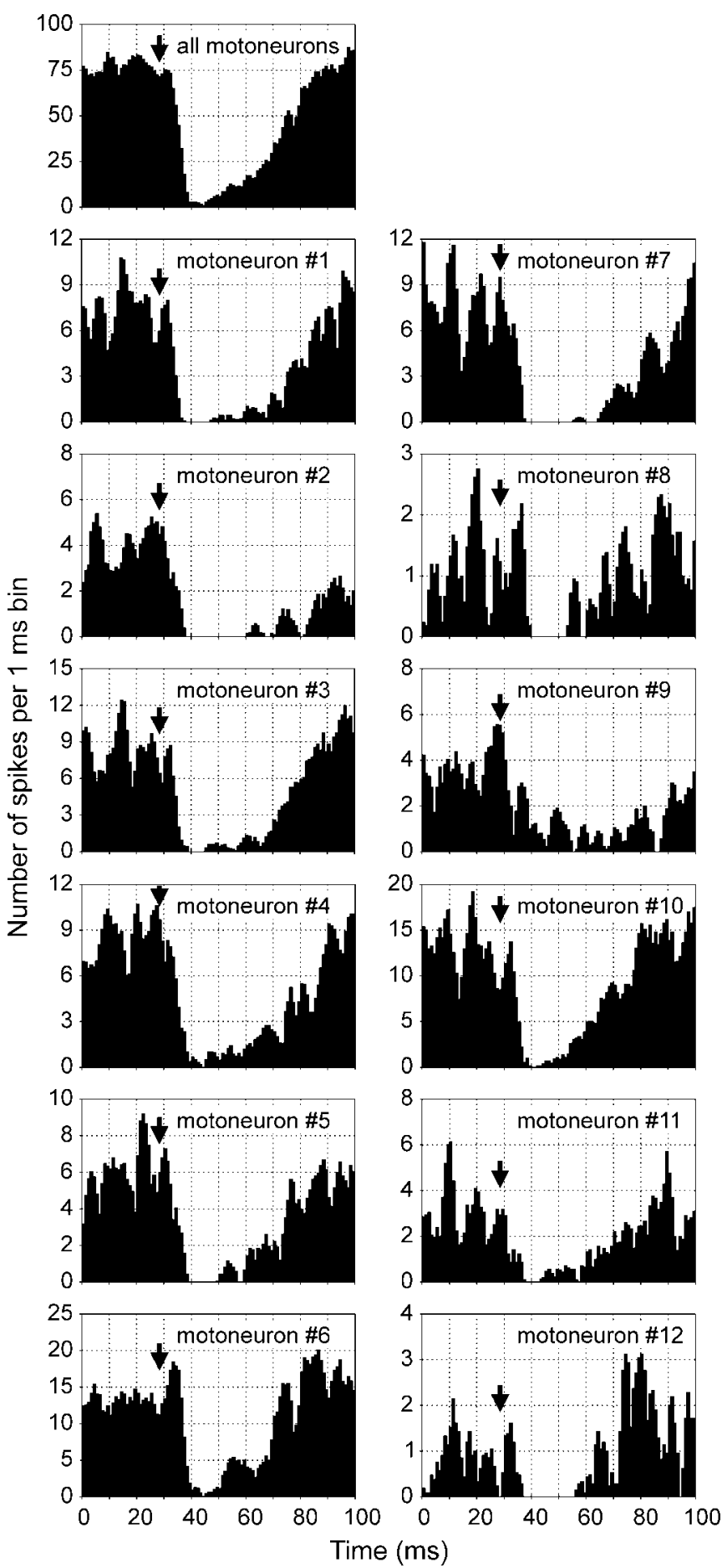

Figure 5. Similarity of influences exerted by an RS neuron on different MNs of the same ventral root branch. A post-RS spike histogram for the whole branch (all motoneurons) and histograms generated separately for 12 MNs (\#1-12) projecting to this branch and discriminated by a spikesorting procedure (see Materials and Methods) are shown.

this effect is combined with excitation of either the contralateral dorsal MNs (F2) or both groups of ipsilateral MNs (F3).

Group $G$ (Fig. 7) adds a ventral component to the ipsilateral flexion and a dorsal component to the contralateral flexion. In the pattern G1, this is caused by excitation of the ipsilateral ventral MNs and inhibition of the contralateral ventral MNs. In the

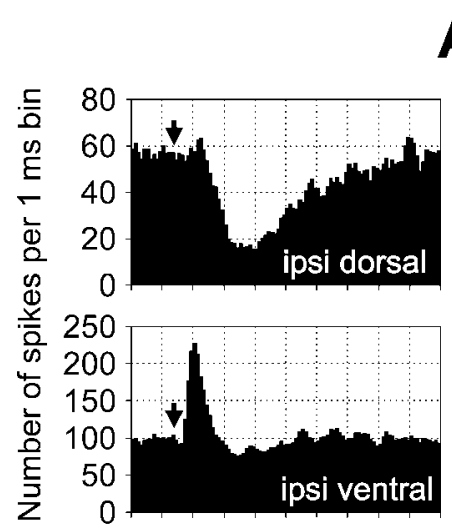

A
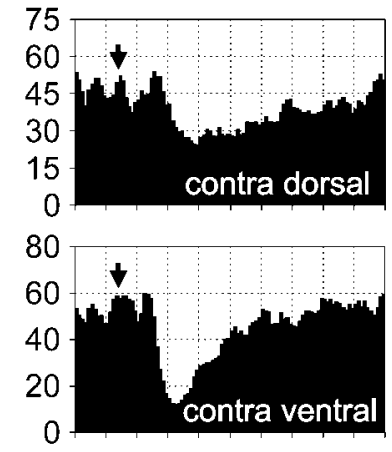

B

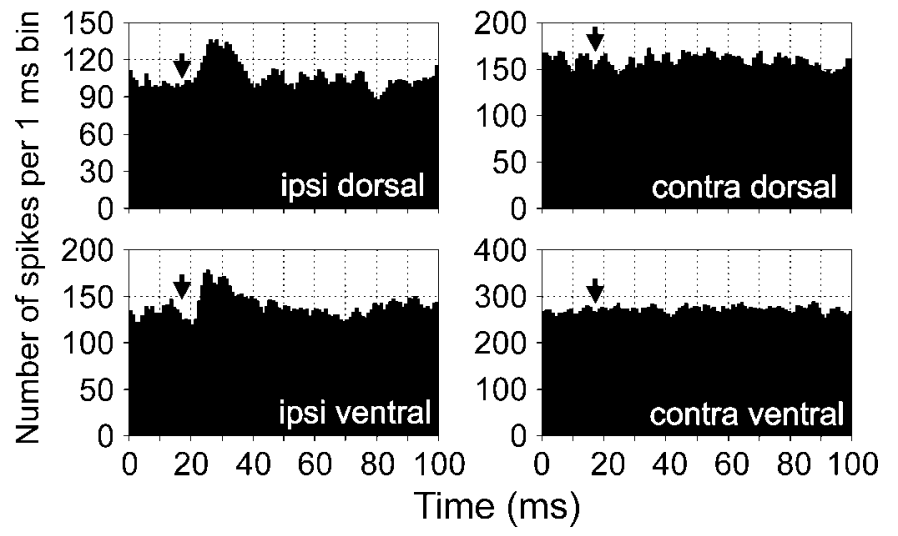

Figure 6. Examples of the patterns of segmental response. A, An RS neuron from MRRN evoked excitation in the ipsilateral ventral branch and inhibition in three other branches-the ipsilateral dorsal, contralateral dorsal, and contralateral ventral. $B$, An RS neuron from PRRN evoked excitation in both ipsilateral branches and did not influence both contralateral branches.

pattern G2, this effect is enhanced by excitation of the contralateral dorsal MNs and inhibition of the ipsilateral dorsal MNs.

In Figure 7, the four numbers under each pattern diagram indicate the numbers of RS neurons with a given pattern recorded from MRN, ARRN, MRRN, and PRRN, respectively. Among 37 RS neurons recorded from MRRN, 18 of 20 patterns of influence on the segmental motor output were present, and only patterns D1 and F2 were absent. In six neurons from MRN, five patterns $(A 3, C 2, D 2, E 2$, and $F 2)$ were present. In seven neurons from ARRN, three patterns $(D 1, E 1$, and E5) were present. Finally, in 10 neurons from PRRN, only two patterns, namely A3 $(n=3)$ and $\mathrm{B} 1(n=7)$ were present.

\section{Longitudinal spinal projections of RS neurons}

The distribution of the RS effects along the spinal cord was studied in a second set of experiments (Fig. 2B). Effects of stimulation of 81 axons were examined in 20 animals. The conduction velocities in these axons ranged from 1.7 to $3.3 \mathrm{~m} / \mathrm{sec}$. Most of the axons $(n=51)$ were located in the medial area of the spinal cord; the rest $(n=30)$ coursed in more lateral areas. A noticeable effect on at least one of the recorded VR branches was observed for 52 of 81 tested axons (64\%).

The basic features of the response pattern persisted in the analogous VR branches at different levels of the spinal cord, as 


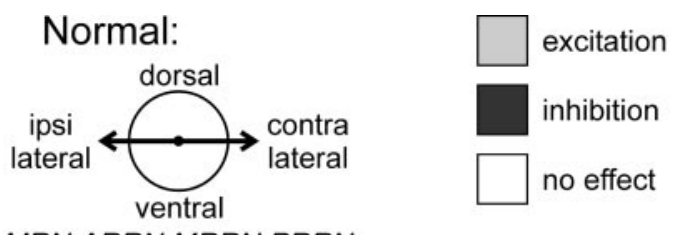

$M R N, A R R N, M R R N, P R R N$

\section{Groups:}
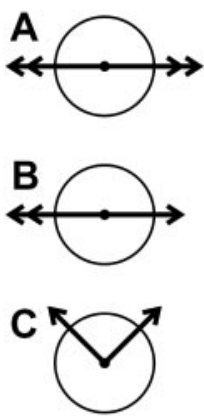

D

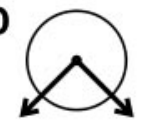

E
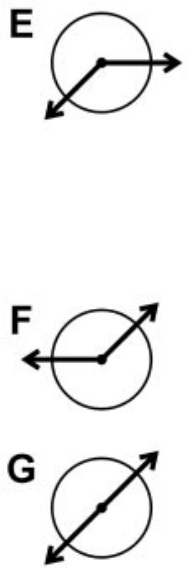

Patterns:
A1

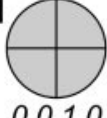

B1

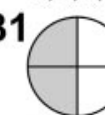

$0,0,5,7$

C1

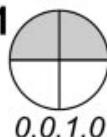

D1

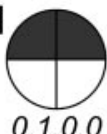

E1

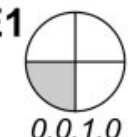

E4

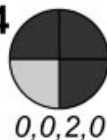

F1

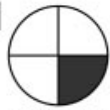

$0,0,1,0$

G1

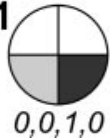

A2

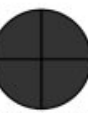

$0,0,2,0$

B2

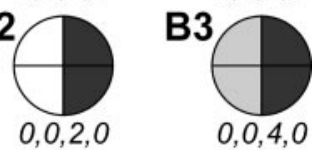

C2

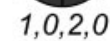

D2

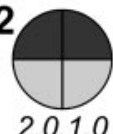

E2

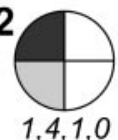

E5

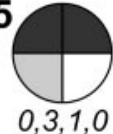

F2

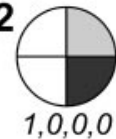

G2
A3

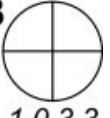

$1,0,3,3$

$0,0,4,0$

E3

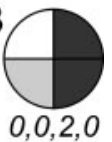

-3

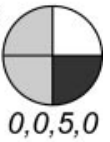

Figure 7. Patterns of segmental responses to stimulation of individual RS neurons. The patterns were defined as the combinations of responses (excitation, inhibition, no effect) in the four VR branches of segment 20. The patterns were classified into seven groups $(A-G)$ according to their presumed effect on the segmental motor output in the two hemicycles of the locomotor cycle, that is, when the ipsilateral MNs are active and when the contralateral MNs are active (an RS neuron is suggested to be active in both hemicycles). The directions of flexion of the segment in these hemicycles are shown by two vectors, respectively. During normal swimming, both vectors are horizontal and of equal magnitude (Normal). Because of the RS influences, each of the two flexions can change its magnitude (denoted by a double arrowhead) or direction (denoted by an inclination of the vector). Four numbers below each diagram show the numbers of RS neurons with a given pattern recorded from a particular reticular nucleus: $M R N, A R R N, M R R N$, and $P R R N$, respectively.

illustrated in Figure $8 A$ for the responses with a dominating excitatory component. This component is clearly seen in all six sites of recording, from segment 13 to segment 83 . The second, inhibitory component is most pronounced in segment 13 and considerably smaller in the other sites of recording.

The effects of an axon with a dominating inhibitory component are illustrated in Figure $8 B$. Again, this component is clearly seen
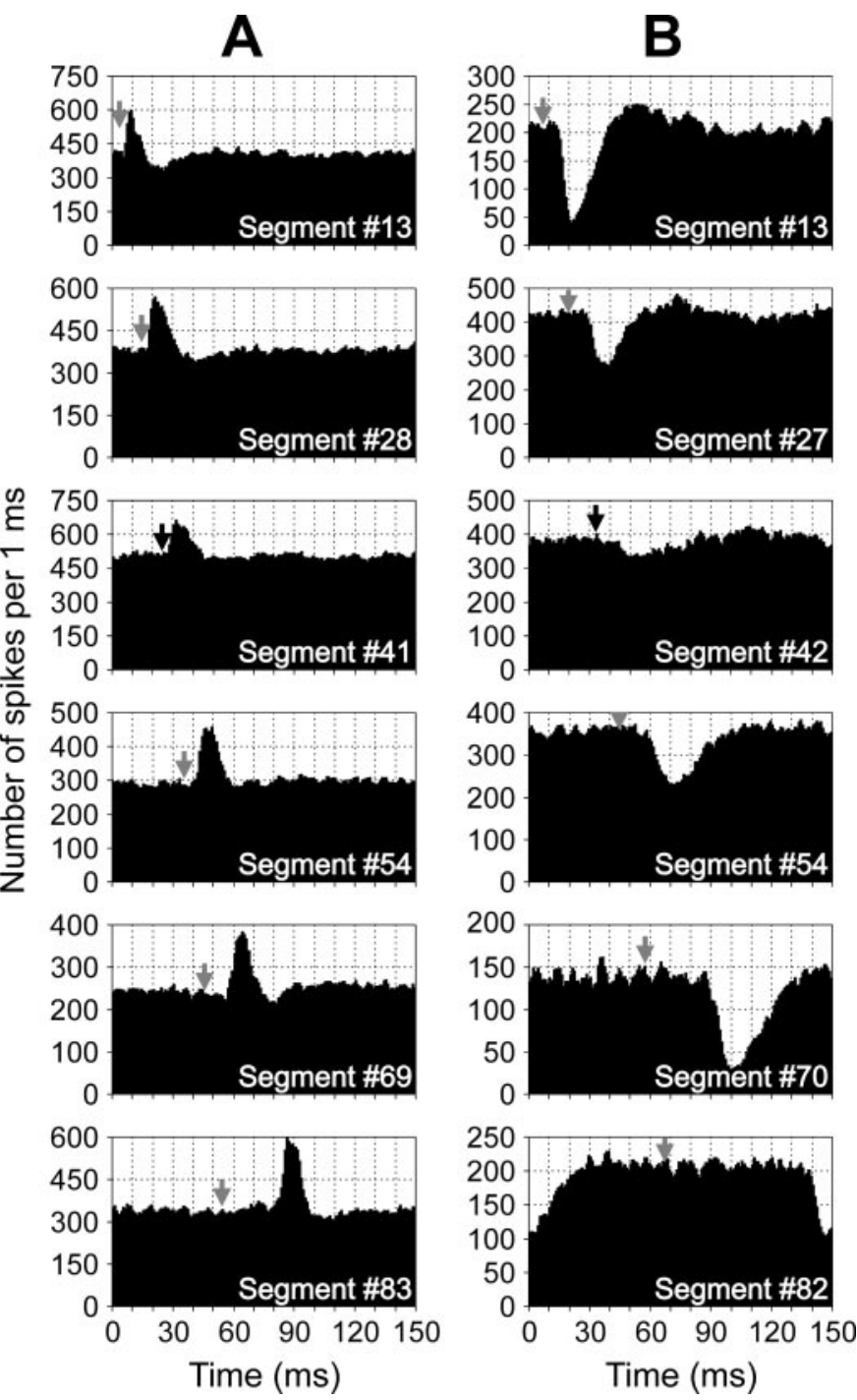

Figure 8. Examples of the RS longitudinal functional projections. A, An $\mathrm{RS}$ axon evoked excitation in the ipsilateral ventral branches. $B$, An RS axon evoked inhibition in the contralateral ventral branches. Black arrows show the moments of the RS spike arrival to segments \#41 and \#42, respectively. Gray arrows show estimated moments of the RS spike arrival to other segments, provided the conduction velocity is constant along the whole extent of the axon. Because the conduction velocities of RS axons in the caudal spinal cord are lower than in the rostral regions (Rovainen, 1982), the segmental delays in the caudal segments are overestimated.

in all six sites of recording, from segment 13 to segment 82 , although the response magnitude in segment 42 is considerably reduced as compared with the other sites of recording. The second, excitatory component is most pronounced in segments 13 and 27 and reduced in all other sites of recording.

To characterize qualitatively the effects of RS neurons, we measured the relative amplitude of the response, i.e., the maximal deviation (in percentage) of the summed MN activity in a VR branch from the level observed before the occurrence of the response. Figure 9 shows the response amplitude at different sites along the spinal cord for 52 influencing $\mathrm{RS}$ axons. The recordings were performed from the ipsilateral dorsal $(A)$, ipsilateral ventral $(B)$, contralateral dorsal $(C)$, and contralateral ventral $(D) \mathrm{VR}$ branches. In the graphs, positive and negative values represent excitatory and inhibitory responses, respectively. The main find- 

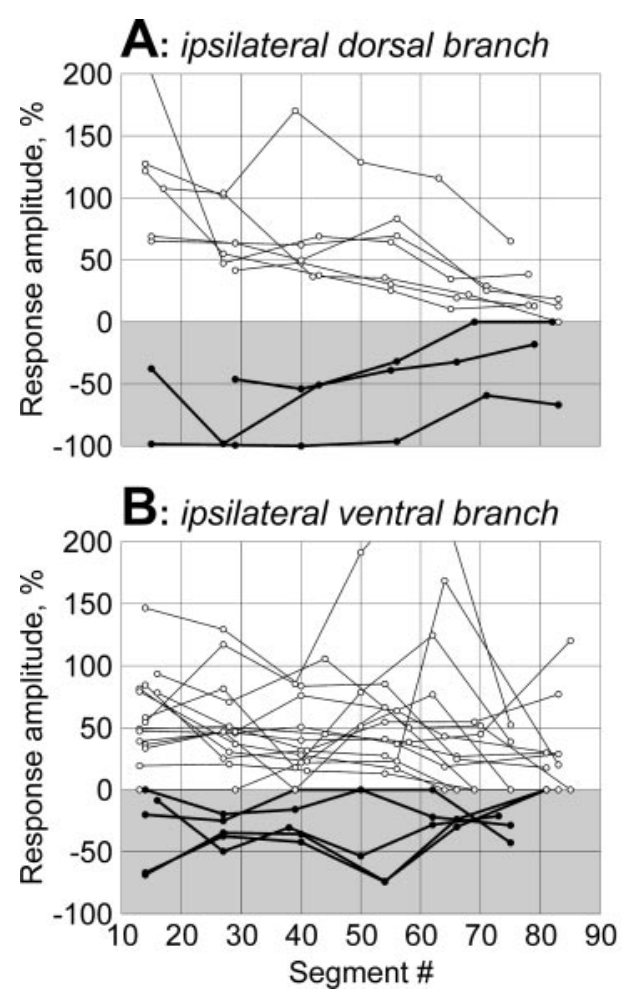
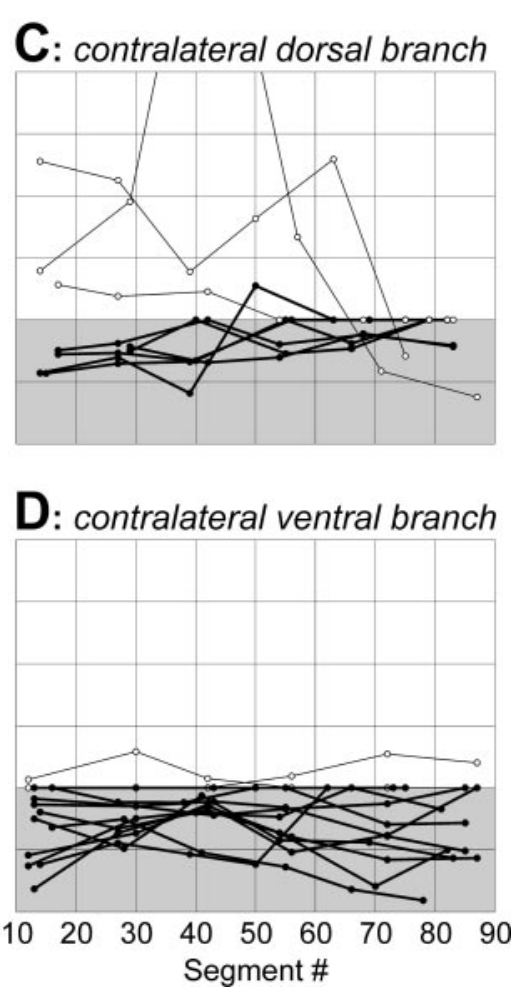

Figure 9. Longitudinal distribution of RS functional projections. Recordings were performed from analogous VR branches: ipsilateral dorsal $(A)$, ipsilateral ventral $(B)$, contralateral dorsal $(C)$, and contralateral ventral $(D)$. For each $\mathrm{RS}$ axon, the relative amplitude of response, that is, the maximal deviation (in percentage) of the summated $\mathrm{MN}$ activity from the level observed before the occurrence of the response, is shown for all sites of recording. Positive and negative values represent the excitatory and inhibitory responses, respectively. The data points for individual axons are connected by lines; thin lines and open circles indicate the axons with exclusively (or prevailing) excitatory influences; thick lines and filled circles indicate those with exclusively (or prevailing) inhibitory influences. ing of this set of experiments is that individual RS neurons exert a uniform action on the locomotor output in different segments along the whole extent of their axons. The sign of the effect changed along the axon in only 3 of 52 axons (Fig. 9C). One can thus conclude that for each individual RS neuron the pattern of effects in the transverse plane found in segment 20 (Fig. 7) is characteristic not only for this particular segment but also for most other segments along the whole extent of the RS axon.

\section{Effects of longitudinal and transverse cuts of the spinal cord}

Long segmental delays of the MN responses (Fig. 4), found in the present study, suggest that RS influences are transmitted to MNs through polysynaptic pathways. To characterize these pathways, experiments with lesions to the spinal cord were performed. The extent of the pathways mediating inhibitory effects on the contralateral MNs was studied in experiments with a longitudinal 15-segment-long midline split of the spinal cord (Fig. 10 $A$ ). (In these experiments, $O$ was assigned to the segment at the rostral end of the split, and 15 was assigned to the segment at the caudal end.) Responses were recorded from the analogous VR branches in six sites, from a site slightly rostral to the split to the site slightly caudal to it. Figure $10 B$ illustrates the effect of the splitting on the $\mathrm{MN}$ responses in different recording sites for one of the RS axons. Rostral to the split (Segment \#-4), the response was of a normal value. At the level of the rostral edge of the split (Segment \#0), the response was markedly reduced. Any response was absent in the middle of the split (Segments \#5 and \#11). At the level of the caudal edge of the split (Segment \#15), a small response was observed. Finally, four segments further caudal (Segment \#19) to the response became larger.

Similar results were obtained in all eight experiments with longitudinal split of the spinal cord, in which 13 axons with contralateral inhibitory influences were examined. These results are summarized in Figure $10 C$, where the response amplitude in different sites is shown. Typically, the responses "penetrate" into the split zone for a distance of only a few segments caudally and $<1$ segment rostrally.

The caudal extent of the interneuronal pathways mediating excitatory and inhibitory RS effects on the ipsilateral MNs was studied in experiments with a transverse cut of the medial area of the spinal cord (Fig. 11A). (In these experiments, \#0 was assigned to the segment at the level of the cut.) With this technique, only the medial RS axons (damaged by the cut) could be examined. Responses were recorded from the analogous VR branches in five sites. The effect of the medial lesion on the MN responses in different recording sites is illustrated in Figure $11 B$ for one of the axons with inhibitory influences. Rostral to the damage (Segment \#-4), the response was of a normal value. At the level of the damage (Segment \#0), the response was markedly reduced. No response was seen caudally to the damage (Segments \#5, \#10, \#20).

Similar results were obtained in all three experiments with the medial cut of the spinal cord, in which nine axons with excitatory influences and six axons with inhibitory influences were examined. These results are summarized in Figure $11 C$, where the amplitude of the response in different sites of recording is shown. The responses were considerably reduced at the level of the lesion and were always absent caudal to the lesion, suggesting a very short effective length of the interneuronal pathways mediating ipsilateral RS influences on MNs.

Analogous experiments with the medial transverse cut of the spinal cord and stimulation of the caudal part of the RS axons (Fig. 11D) showed that the rostral extent of the interneuron pathways mediating the ipsilateral influences is approximately one segment, because none of the five axons with excitatory influences and none of the three axons with inhibitory influences produced any effect rostral to the cut (Fig. 11E). 


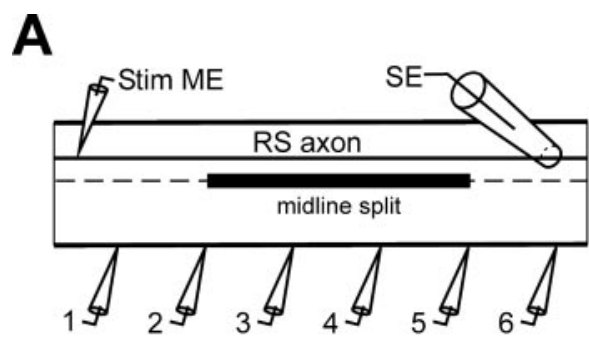

\section{C: extent of contralateral effects}

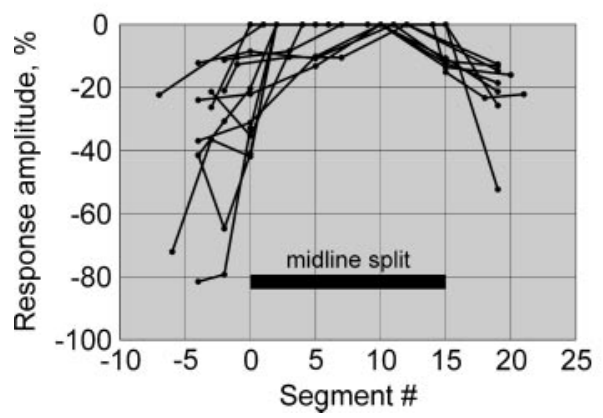

B

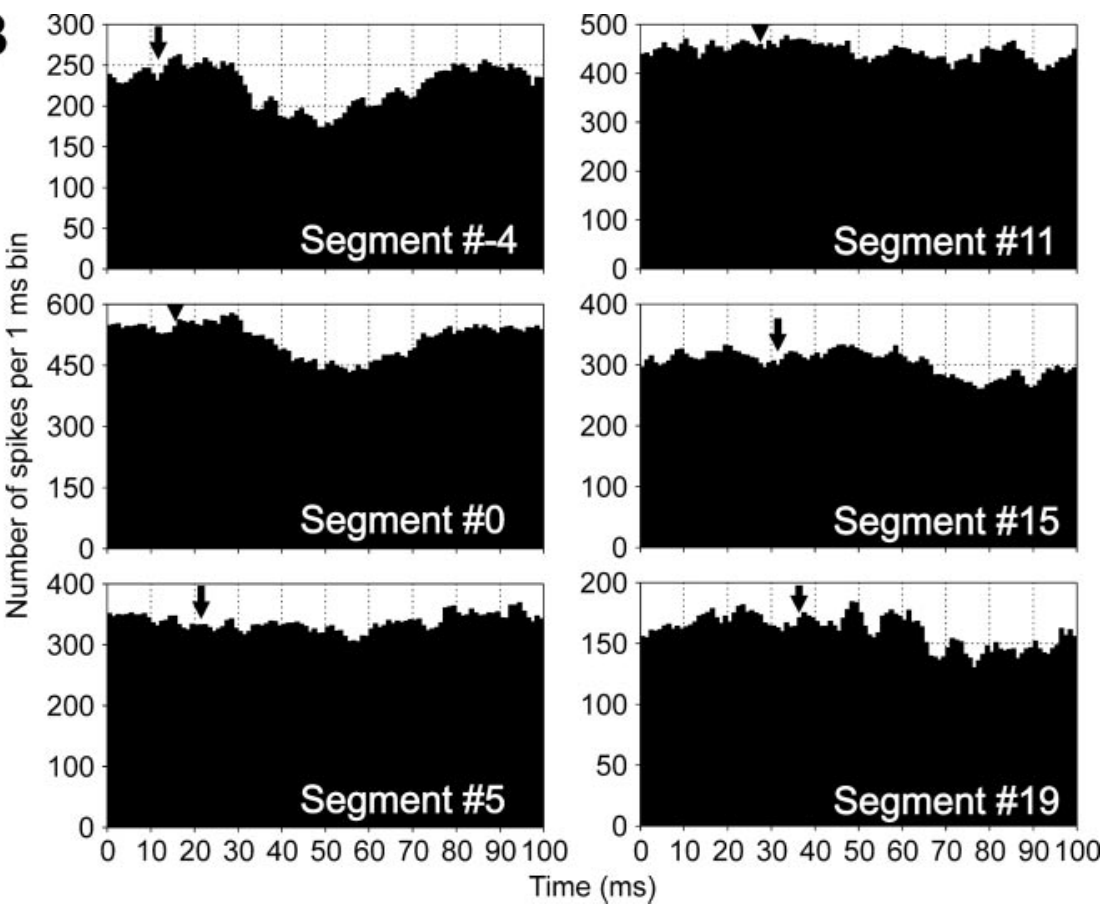

Figure 10. Effective length of interneuronal pathways transmitting inhibitory RS influences to contralateral MNs. $A$, Experimental design. An RS axon was stimulated through the intracellular electrode (Stim ME). Axons of commissural interneurons were cut by a 15-segment-long midline split. Activity of MNs was recorded from six analogous ventral root branches contralateral to the RS axon with suction electrodes (1-6). A propagating spike in the $\mathrm{RS}$ axon was recorded by a surface electrode $(S E)$. B. An example of the effects of midline split on MN responses in different sites of recording. This particular RS axon evoked inhibition in the contralateral ventral VR branches. $C$, Summary of the midline split effects on the contralateral RS influences. For each RS axon, the relative amplitude of response is shown for all sites of recording. Negative values represent inhibitory responses. The data points for individual axons are connected by lines. Location of the midline split is indicated. In $B$ and $C$, \# 0 was assigned to the segment at the rostral end of the split.

\section{DISCUSSION}

In the present study, the effects of individual reticulospinal command neurons on numerous outputs of the spinal locomotor networks have been characterized for the first time. This study has shown that each RS neuron exerts a uniform effect on the segmental motor output along the whole extent of its axon (Fig. 9). Various effects, defined as combinations of influences on the four MN pools in a segment, have been found (Fig. 7). The capacity of RS neurons to evoke flexion of a considerable part of the body in a certain plane is essential for the control of steering and postural orientation (Ullén et al., 1995; McClellan and Hagevik, 1997). One can suggest that for a turn in a certain plane, a specific group of RS neurons, with the corresponding pattern of influences on the spinal networks, has to be activated. Group B might cause lateral turns, whereas groups $\mathrm{C}$ and $\mathrm{D}$ might cause turns in the sagittal plane, up and down, respectively. Finally, groups $\mathrm{E}-\mathrm{G}$, eliciting a deviation of the plane of locomotor undulations from the normal (horizontal) one, might cause roll turns.

The most likely role of the RS neurons with pattern A1 (activation of all four segmental outputs) and pattern A2 (inhibition of all four segmental outputs) is a regulation of the amplitude of locomotor undulations during rectilinear swimming. However, among the 60 recorded RS neurons, only 1 neuron with pattern A1 and 2 neurons with pattern A2 were found. One can suggest that an important function, i.e., the elicitation and control of rectilinear swimming, is performed through simultaneous activation of RS neurons with "opposite asymmetry," e.g., the neurons with patterns B1 and B2 on the left and right sides.
The presumed motor effects of different groups of RS neurons considered above were derived on the basis of the supposition that these neurons are active in both hemicycles of the locomotor cycle, that is, when the ipsilateral and contralateral MNs are active (Fig. $2 C$ ). It is known, however, that the activity of a large proportion of RS neurons in the lamprey is rhythmically modulated in relation to the locomotor cycle. This modulation is caused by the internal feedback signals coming from the spinal locomotor networks (Dubuc and Grillner, 1989; Kasicki et al., 1989; Deliagina et al., 2000); however, the functional role of this modulation has not been clarified. A certain role can be attributed to the modulation based on results of the present study. Depending on the phase of activity of an RS neuron in the locomotor cycle, its motor effect can change dramatically. For example, the RS neuron with pattern A1 (Fig. 7) will cause no turn when activated tonically; it will cause an ipsilateral turn when activated in phase with the ipsilateral hemisegment and a contralateral turn when activated in phase with the contralateral hemisegment. Thus, the phase-dependent modulation of RS neurons will lead to a further increase of the diversity of their influence patterns.

The diversity of patterns of functional spinal projections differed strongly between the two main reticular nuclei, MRRN and PRRN. In MRRN, 18 of the total number of 20 patterns were present. By contrast, among the PRRN neurons that exerted any influence on the segmental motor output, only one pattern, the ipsilateral excitation (Fig. 7, pattern B1), was present. This corresponds to earlier findings that the MRRN neurons receive powerful sensory inputs, suggesting their important role in the control of posture and steering (Deliagina et al., 1993; Ullén et 

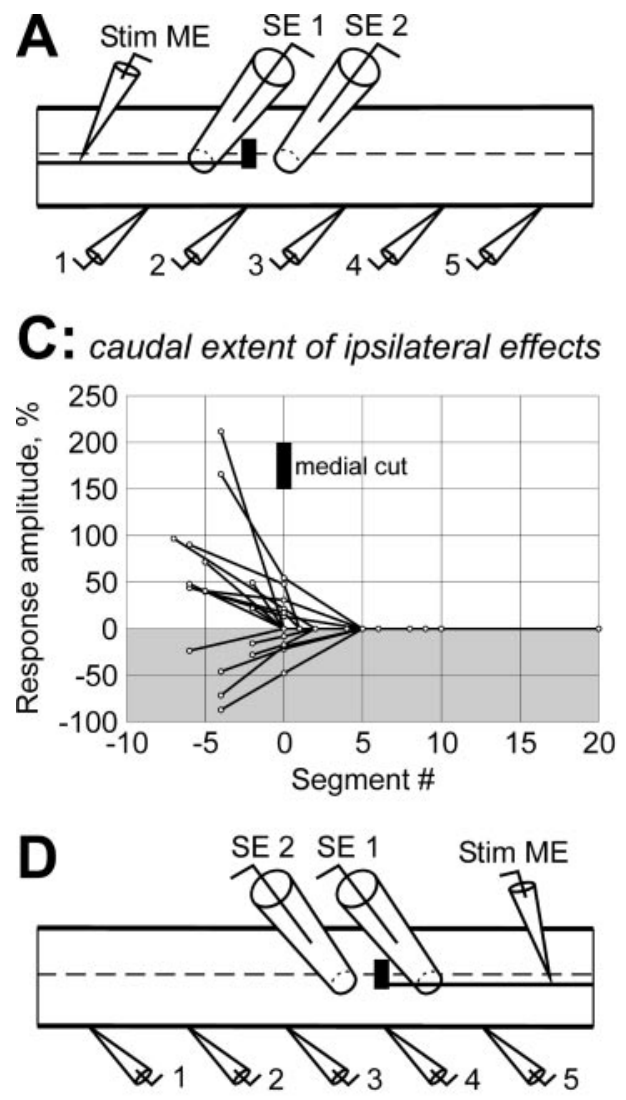

\section{E: rostral extent of ipsilateral effects}

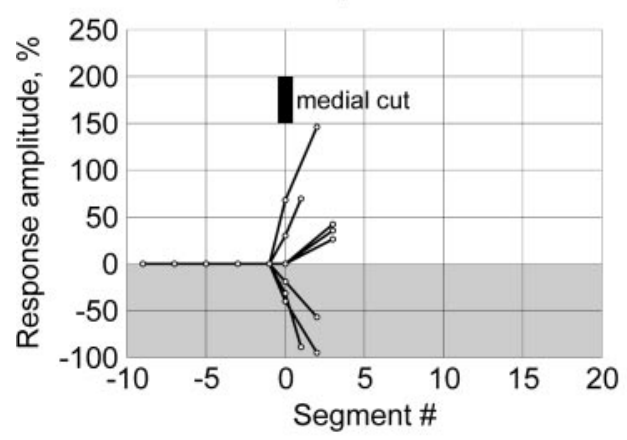

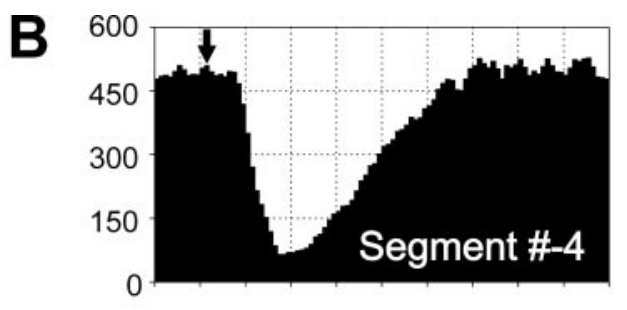
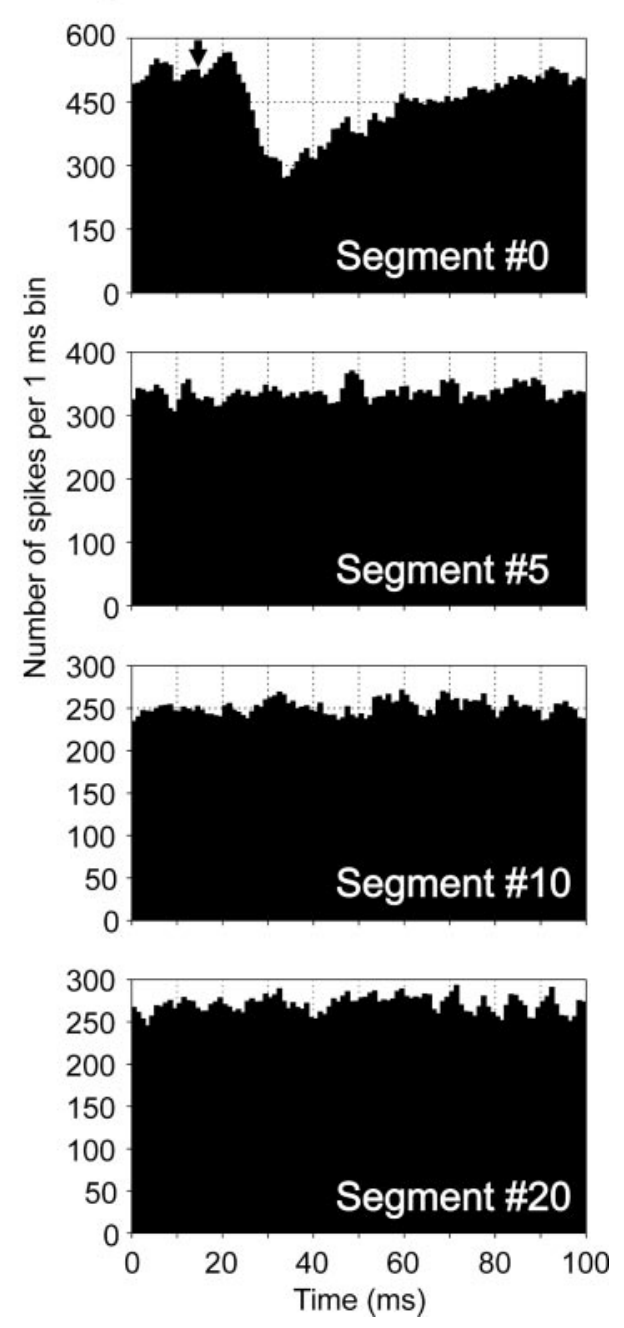

Figure 11. Effective length of interneuronal pathways transmitting $\mathrm{RS}$ influences to ipsilateral MNs. $A-C$, Transmission of descending influences. $A$, Experimental design. Medial RS axons were transected by a medial cut. Rostral part of the RS axon was stimulated through the intracellular electrode (Stim ME). Activity of MNs was recorded from five analogous VR branches (1-5). A propagating spike in the RS axon was recorded by a surface electrode ( $S E 1)$ positioned rostrally to the lesion. An electrode ( $S E$ 2), positioned caudally to the lesion, was used to confirm transection of the axon. $B$, An example of the effects of medial cut on $\mathrm{MN}$ responses in different sites of recording. This particular RS axon evoked inhibitory responses in the ipsilateral ventral VR branches. $C$, Summary of the medial cut effects on the ipsilateral descending RS influences. Gray background is used for inhibitory responses; white background is used for excitatory ones. $D, E$, Transmission of ascending influences. $D$, Experimental design. Medial RS axons were transected by a medial cut. Caudal part of the RS axon was stimulated through the intracellular electrode (Stim ME). Activity of MNs was recorded from five analogous VR branches (1-5). A propagating spike in the RS axon was recorded by a surface electrode (SE 1) caudally to the lesion. An electrode ( $S E$ $2)$, positioned rostrally to the lesion, confirmed transection of the axon. $E$, Summary of the medial cut effects on the ipsilateral ascending RS influences. In $B-E, \# 0$ was assigned to the segment where the medial cut was made. al., 1996). The most likely function of the PRRN neurons is an initiation of locomotion (Wannier et al., 1998); they may also participate in the control of lateral turns (Ullén et al., 1998).

Spikes in an RS axon, when arriving regularly, will affect the mean frequency of MNs. This effect depends on the spike frequency. In intact lampreys, a characteristic firing frequency of RS neurons is $\sim 10 \mathrm{~Hz}$. (Deliagina and Fagerstedt, 2000; Deliagina et al., 2000). This frequency was also used in the present study when stimulating RS neurons. To calculate a change in the mean MN frequency caused by an "average" RS neuron, we approximated an $\mathrm{MN}$ response by a triangle, with a base equal to the average response duration and the height equal to the average response amplitude in percentage. The area of the triangle was then divided by the area of the rectangle ("background activity") with the height equal to $100 \%$ and the base equal to $100 \mathrm{msec}$ (the interval between RS spikes at $10 \mathrm{~Hz}$ ). We found that an average excitatory RS neuron will cause an increase of the mean activity of MNs by $11 \%$, whereas an average inhibitory RS neuron will cause a decrease by $15 \%$. Thus, a contribution of single RS neurons to the generation of spinal motor output in the lamprey is significant, at least at relatively low levels of activity in the spinal network. With an increase of this activity, this contribution will most likely decrease. One has also to take into account that we examined the effects of only large and middle-size RS neurons. The effects of smaller neurons, constituting the main part of the RS system (Bussières, 1994), may be weaker.

In higher vertebrates, influences of single RS neurons on the motor output are difficult to reveal even with the technique of spike-triggered averaging (Drew et al., 1986), and information about RS projections comes mainly from anatomical studies and from experiments with stimulation that activates a sufficient number of neurons to produce a noticeable motor effect. Anatomical studies have shown that individual RS neurons may project to one, two, three, or even four spinal limb centers (Matsuyama et al., 1997; Peterson, 1979). It remains unclear, however, whether all these widespread projections are effective ones and what 
effects on the motor output they produce. Experiments with electrical stimulation of the reticular formation have shown that the RS system in higher vertebrates differs dramatically from other descending systems (e.g., corticospinal) in that it has almost no somatotopic organization: stimulation of relatively small areas evoked widespread motor effects (Magoun, 1950). This peculiarity of the RS system led to a suggestion that this system has no firm functional differentiation (Siegel, 1979). More recently, by stimulation of even smaller areas, some specificity in RS influences on different limbs and different muscular groups was demonstrated (Drew and Rossignol, 1990a,b; Drew, 1991). A low specificity of RS influences revealed in the experiments with stimulation was most likely caused by simultaneous activation of a group of RS neurons with different specific projections. As shown in the present study, even the neurons from the same reticular nucleus in the lamprey have a rich variety of functional projection patterns.

Long segmental delays were observed for both excitatory and inhibitory RS influences on the contralateral MNs, as well as for the inhibitory ipsilateral influences (Fig. 4), suggesting polysynaptic transmission of these influences. Experiments with lesions to the spinal cord allowed us to estimate the effective length of propriospinal pathways mediating RS influences. Experiments with a midline split have shown that the characteristic distance for propagation of contralateral inhibitory influences is a few segments caudal and even less rostral (Fig. 10C), suggesting that these influences are mediated by the commissural interneurons with the corresponding length of their axons. The likely candidates for this role are the short-axon commissural interneurons (Ohta et al., 1991) but not the long-axon ones (Buchanan, 1982).

Experiments with medial cuts of the spinal cord have shown that ipsilateral excitatory and inhibitory influences propagate rostrally and caudally for less than one segment (Fig. 11C,E), suggesting that the interposed interneurons have a corresponding short length of their axons. The likely candidates for these roles are the ipsilaterally projecting short-axon excitatory interneurons (Buchanan et al., 1989) and inhibitory interneurons (Buchanan and Grillner 1988) but not the long-axon interneurons (Rovainen, 1974a). Interestingly, an important role was also assigned to the short-axon propriospinal neurons for the locomotor rhythm generation, for intersegmental coupling (Buchanan, 1999), and for crossed reciprocal inhibition (Fagerstedt et al., 2000).

In conclusion, two principal findings of the present study, namely (1) widespread effects of individual RS neurons and (2) a rich variety of behaviorally reasonable projection patterns, strongly suggest that RS neurons are responsible for the formation of motor synergies underlying control of rectilinear swimming as well as turns in different planes.

\section{REFERENCES}

Buchanan JT (1982) Identification of interneurons with contralateral, caudal axons in the lamprey spinal cord: synaptic interactions and morphology. J Neurophysiol 47:961-975.

Buchanan JT (1999) Commissural interneurons in rhythm generation and intersegmental coupling in the lamprey spinal cord. J Neurophysiol 81:2037-2045.

Buchanan JT, Cohen AH (1982) Activities of identified interneurons, motoneurons, and muscle fibers during fictive swimming in the lamprey and effects of reticulospinal and dorsal cell stimulation. J Neurophysiol 47:948-960.

Buchanan JT, Grillner S (1988) A new class of small inhibitory interneurons in the lamprey spinal cord. Brain Res 438:404-407.
Buchanan JT, Grillner S, Cullheim S, Risling M (1989) Identification of excitatory interneurons contributing to generation of locomotion in lamprey: structure, pharmacology, and function. J Neurophysiol 62:59-69.

Bussières N (1994) Les systemes descendants chez la lamproie. Etude anatomique et functionnelle. Montreal: University of Montreal.

Camp C, Pinsker H (1979) Computer separation of unitary spikes from whole-nerve recordings. Brain Res 169:455-479.

Currie SN, Carlsen RC (1987) Functional significance and neural basis of larval lamprey startle behaviour. J Exp Biol 133:121-135.

Deliagina T, Fagerstedt P (2000) Responses of reticulospinal neurons in intact lamprey to vestibular and visual inputs. J Neurophysiol 83:864-878.

Deliagina TG, Orlovsky GN, Grillner S, Wallén P (1992) Vestibular control of swimming in lamprey. 2. Characteristics of spatial sensitivity of reticulospinal neurons. Exp Brain Res 90:489-498.

Deliagina TG, Grillner S, Orlovsky GN, Ullén F (1993) Visual input affects the response to roll in reticulospinal neurons of the lamprey. Exp Brain Res 95:421-428.

Deliagina T, Zelenin P, Fagerstedt P, Grillner S, Orlovsky G (2000) Activity of reticulospinal neurons during locomotion in the freely behaving lamprey. J Neurophysiol 83:853-863.

Drew T (1991) Functional organization within the medullar reticular formation in intact unrestrained cat. III. Microstimulation during locomotion. J Neurophysiol 66:919-938.

Drew T, Rossignol S (1990a) Functional organization within the medullary reticular formation in intact unrestrained cat. I. Movements evoked by microstimulation. J Neurophysiol 64:767-781.

Drew T, Rossignol S (1990b) Functional organization within the medullary reticular formation in intact unrestrained cat. II. Electromyographic activity evoked by microstimulation. J Neurophysiol 64:782-795.

Drew T, Dubuc R, Rossignol S (1986) Discharge patterns of reticulospinal and other reticular neurons in chronic, unrestrained cats walking on a treadmill. J Neurophysiol 55:375-401.

Dubuc R, Grillner S (1989). The role of spinal cord inputs in modulating the activity of reticulospinal neurons during fictive locomotion in the lamprey. Brain Res 483:196-200.

Fagerstedt P, Zelenin PV, Deliagina TG, Orlovsky GN, Grillner S (2000) Crossed reciprocal inhibition evoked by electrical stimulation of the lamprey spinal cord. Exp Brain Res 134:147-154.

Fetz EE, Cheney PD (1980) Postspike facilitation of forelimb muscle activity by primate corticomotoneuronal cells. J Neurophysiol 44:751-772.

Grillner S, McClellan A, Sigvardt K, Wallén P, Wilén M (1981) Activation of NMDA-receptors elicits "fictive locomotion" in lamprey spinal cord in vitro. Acta Physiol Scand 113:549-551.

Grillner S, Deliagina T, Ekeberg O, el Manira A, Hill RH, Lansner A, Orlovsky GN, Wallén P (1995) Neural networks controlling locomotion and body orientation in lamprey. Trends Neurosci 18:270-279.

Kasicki S, Grillner S, Ohta Y, Dubuc R, Brodin L (1989) Phasic modulation of reticulospinal neurones during fictive locomotion and other types of spinal motor activity in lamprey. Brain Res 484:203-216.

Liu KS, Fetcho JR (1999) Laser ablations reveal functional relationships of segmental hindbrain neurons in zebrafish. Neuron 23:325-335.

Magoun HW (1950) Caudal and cephalic influences of the brain stem reticular formation. Physiol Rev 30:459-474.

Matsuyama K, Takakusaki K, Nakajima K, Mori S (1997) Multisegmental innervation of single pontine reticulospinal axons in the cervico-thoracic region of the cat: anterograde PHA-L tracing study. J Comp Neurol 377:234-250.

McClellan AD, Hagevik A (1997) Descending control of turning locomotor activity in larval lamprey: neurophysiology and computer modeling. J Neurophysiol 78:214-228.

Nieuwenhuys R (1972) Topological analysis of the brain stem of the lamprey Lampetra fluviatilis. J Comp Neurol 145:165-177.

Nieuwenhuys R, ten Donkelaar HR, Nicholson C (1996) The central nervous system of vertebrates. Berlin: Springer.

Ohta Y, Grillner S (1989) Monosynaptic excitatory amino acid transmission from the posterior rhombencephalic reticular nucleus to spinal neurons involved in the control of locomotion in lamprey. J Neurophysiol 62:1079-1089.

Ohta Y, Dubuc R, Grillner S (1991) A new population of neurons with crossed axons in the lamprey spinal cord. Brain Res 564:143-148.

Orlovsky GN, Deliagina TG, Grillner S (1999) Neuronal control of locomotion. From mollusc to man. Oxford: Oxford UP.

Peterson BW (1979) Reticulospinal projections to spinal motor nuclei. Annu Rev Physiol 41:127-140.

Ronan M (1989) Origins of the descending spinal projections in petromyzontid and myxinoid agnathans. J Comp Neurol 281:54-68.

Rovainen CM (1967) Physiological and anatomical studies on large neurons of central nervous system of the sea lamprey (Petromyzon marinus). I. Muller and Mautner cells. J Neurophysiol 30:1000-1023.

Rovainen CM (1974a) Synaptic interactions of identified nerve cells in the spinal cord of the sea lamprey. J Comp Neurol 154:189-206. 
Rovainen CM (1974b) Synaptic interactions of reticulospinal neurons and nerve cells in the spinal cord of the sea lamprey. J Comp Neurol 154:207-224.

Rovainen CM (1979) Electrophysiology of vestibulospinal and vestibuloreticulo-spinal systems in lampreys. J Neurophysiol 42:745-766.

Rovainen CM (1982) Neurophysiology. In: The biology of lampreys, Vol 4A (Hardisty MW, Potter IC, eds), pp 37-40. London: Academic.

Siegel JM (1979) Behavioral functions of the reticular formation. Brain Res Rev 1:69-105.

Tretjakoff D (1927) Das nervensystem des flussnevnauges. Z Wiss Zool 129:359-452.

Ullén F, Deliagina TG, Orlovsky GN, Grillner S (1995) Spatial orientation of lamprey. 1. Control of pitch and roll. J Exp Biol 198:665-673.
Ullén F, Deliagina T, Orlovsky G, Grillner S (1996) Visual potentiation of vestibular responses in lamprey reticulospinal neurons. Eur J Neurosci 8:2298-2307.

Ullén F, Fagerstedt P, Ekeberg Ö, Lansner A, Orlovsky GN, Grillner S (1998) Lateral turns in the lamprey: II. The brain descending command system-computer modeling and lesion studies. Soc Neurosci Abstr 24:1156.

Wannier T, Deliagina TG, Orlovsky GN, Grillner S (1998) Differential effects of reticulospinal system on locomotion in lamprey. J Neurophysiol 80:103-112.

Zelenin PV, Orlovsky GN, Grillner S, Deliagina TG (2000) Motor effects of individual reticulospinal neurons in lamprey. Soc Neurosci Abstr 26:1234. 